\title{
A Retrospective Evaluation of Mean Platelet Volume and the Neutrophil-to-lymphocyte Ratio in Children with Chronic Urticaria
}

\author{
(1) Mehmet Ali Duman1, (1) Hatice Duman², (1) Ilteriş Oğuz Topal2, (1) Hatice Nilgün Selçuk Duru¹ \\ 1istanbul Haseki Training and Research Hospital, Clinic of Pediatrics, İstanbul, Turkey \\ 2istanbul Okmeydanı Training and Research Hospital, Clinic of Dermatology, İstanbul, Turkey
}

\begin{abstract}
Aim: Recently, it has been reported that the mean platelet volume (MPV) and neutrophil-to-lymphocyte ratio (NLR) can be used as indicators of inflammation in various inflammatory diseases such as psoriasis, ankylosing spondylitis and ulcerative colitis. In this study, we aimed to assess MPV levels and NLR in children with chronic urticaria (CU) and to compare them with healthy controls.

Materials and Methods: The cases of 46 children with CU [11 with inducible urticaria (IU) and 35 with chronic spontaneous urticaria (CSU)] admitted to our outpatient clinics, and 30 healthy children were evaluated retrospectively.

Results: No statistically significant differences among the CU, CSU, and IU and the control groups were found in terms of NLR and MPV levels $(p>0.05)$. The platelet counts in the CU, CSU, and IU and control groups were not statistically significantly different ( $p>0.05)$.

Conclusion: We found that MPV levels and NLR were not different in children with CU, CSU, and IU and in healthy children. It seems that the MPV and NLR cannot be used as inflammatory markers of CU in children. We believe that further studies are required in this field.

Keywords: Chronic urticaria, mean platelet volume, neutrophil-to-lymphocyte ratio
\end{abstract}

\section{Introduction}

Chronic urticaria (CU) is an inflammatory disease, characterized by a daily or near daily occurrence of urticarial symptoms, occurring over a period of more than 6 weeks. $\mathrm{CU}$ in children is less common than in adults, affecting $0.1-3.0 \%$ of children $(1,2)$. CU is divided into 2 sub-types, known as chronic spontaneous urticaria (CSU) and inducible urticaria (IU). Unlike CSU, in IU, the appearance of clinical manifestations is evoked by physical-environmental stimuli and not spontaneously. The two subtypes of CU can coexist within the same child (3).
The pathogenesis of $\mathrm{CU}$ is complex and not yet fully understood. However, mast cells are the key elements in $\mathrm{CU}$ pathogenesis $(4,5)$. $\mathrm{CU}$ is characterized by predominantly non necrotizing infiltrate CD4+ lymphocytes, with variable numbers of monocytes, neutrophils, eosinophils, and basophils as an inflammatory disease of skin $(6,7)$. Platelets play a crucial role in inflammation and their role in $\mathrm{CU}$ has been investigated (8). Platelet size indices, neutrophilto-lymphocyte ratio (NLR) and C-reactive protein (CRP) have been reported as an inflammatory index in various inflammatory diseases such as urticaria, psoriasis and 
Familial Mediterranean fever (FMF) (7-10). Studies assessing the association of CU and mean platelet volume (MPV) in children, however, are limited $(8,11)$. To our knowledge, NLR has not so far been evaluated in patients with urticaria. In this study, we aimed to assess MPV levels and NLR in children with CU and to compare them with those of healthy children.

\section{Materials and Methods}

The cases of 46 children with CU who were 18 years of age or less ( 11 with IU and 35 with CSU) admitted to our outpatient clinics between April 2013 and August 2015 were evaluated retrospectively. Because of the retrospective nature of this study, Haseki Training and Research Hospital Local Ethics Committee rejected to our application. An informed consent form was obtained from the patients' parents.

Those children with CSU with active lesions were included in the study. Those children who had a history of diseases such as hypertension, hyperlipidemia, obesity, diabetes mellitus (DM) that affect MPV levels and those who had a history of any corticosteroids use within one month and antihistamines use within one week prior to admission were excluded from the study (12). Age and sex-matched healthy children who visited the outpatient clinics for regular checkups were enrolled into the control group. The control group included 30 healthy children who were 18 years of age or less, with no history of any dermatological and systemic diseases, and had no history of any drug use within 6 months prior to the study. NLR was obtained by dividing the neutrophil count by the lymphocyte count. The age, age at onset, duration of disease, gender, presence of any concomitant disease, leukocyte, neutrophil, lymphocyte, platelet, MPV, NLR, and CRP levels of the children with CU were examined. In addition, routinely requested tests for diseases that may accompany or may trigger CU (complete blood count, liver and kidney function tests, urine analysis, thyroid function tests and antibodies, total immunoglobulin $\mathrm{E}$, throat and urine cultures, stool parasite exam, anti-streptolysin $\mathrm{O}$, hepatitis markers (hepatitis B surface antigen ( $\mathrm{HBsAg}$ ), antiHBs, anti-hepatitis C virus), Helicobacter pylori (H. pylori) stool direct antigen, anti-nuclear antibodies, rheumatoid factor, lung and sinus X-ray) were examined. In the control group, the age, gender, leukocyte, neutrophil, lymphocyte, platelet, MPV, and NLR levels were examined and compared with those of the children with CU.

\section{Statistical Analysis}

SPSS 15.0 for Windows was used for the statistical analysis. For descriptive statistics; numbers and percentages for categorical variables, and the mean and standard deviation, and the median for quantitative variables were given. Comparisons between two independent groups were carried out using the Student's t-test for quantitative variables with normal distribution, and the Mann-Whitney $U$ test was used for quantitative variables which did not exhibit normal distribution. For comparisons of more than 2 groups, the one-way ANOVA test was used when the normal distribution condition was present, and the Kruskal-Wallis test was used when the parametric test condition was not present. The rates of categorical variables between groups were tested by chi-square analysis. When the relationship between the numerical variables provided parametric test conditions, the Pearson correlation analysis was applied, when it did not provide results, the Spearman correlation analysis was used. Statistical significance was accepted as $p<0.05$.

\section{Results}

Of the 46 children with CU, 18 (39.1\%) were girls and 28 (60.9\%) were boys; of the 30 healthy children, 10 (33.3\%) were girls and $20(66.7 \%)$ were boys. The mean age of the children with CU was $12.59 \pm 4.55$ years ( $3-18$ years), and of the control group, $11.5 \pm 3.1$ years ( $5-15$ years). The two groups were matched in terms of mean age and gender ( $p>0.05)$. The mean age at disease onset was $11.35 \pm 5.20$ years (1-18 years). The duration of the disease was $18.67 \pm 27.68$ months (1.5-144 months).

Thirty-two children had CSU, 8 had symptomatic dermographism, 2 had cold urticaria, 2 had CSU + cholinergic urticaria, 1 had CSU+cold urticaria, and 1 had symptomatic dermographism + cold urticaria in the CU patient group. Three children with both CSU and IU were included in the CSU group. According to this, 11 (23.9\%) of children had IU, and 35 (76.1\%) had CSU.

Four children with CU were concomitant as follows: one of the children had psoriasis, 1 had vitiligo, 1 had FMF, and 1 had chronic sinusitis. The results of $H$. pylori stool direct antigen of 25 children with CU were obtained. Eleven of twenty-five children with CU were positive for $\mathrm{H}$. pylori stool direct antigen. According to these findings, concomitant and other chronic inflammatory conditions (accompanying other chronic inflammatory disease and $H$. pylori stool direct antigen positivity) related to $\mathrm{CU}$ were present in a total of 13 children.

No statistically significant difference was detected between the CSU, IU and control groups in terms of leukocyte, hemoglobin $(\mathrm{Hg})$, neutrophil, lymphocyte, 
platelet counts, NLR and MPV mean values ( $p>0.05)$. No statistically significant difference was detected between the $\mathrm{CU}$ group and the control group's leukocyte, $\mathrm{Hg}$, neutrophil, lymphocyte, platelet counts, NLR, and MPV mean values ( $p>0.05)$ (Table I).

No statistically significant difference was found in CRP levels between the children with IU and CSU ( $p>0.05)$.

In the $\mathrm{CU}$ and control groups, the platelet counts were negatively correlated with MPV levels $(r=-0.345, p=0.002$; $r=-0.474, p=0.008$ ).

There was not any statistically significant difference between CRP levels, platelet counts, MPV levels and NLR of children with $\mathrm{CU}$ with $H$. pylori positive and the children with $\mathrm{CU}$ with $H$. pylori negative $(n=25, p>0.05)$. No statistically significant difference in platelet counts, MPV levels and NLR were found between the children with $\mathrm{CU}$ with $\mathrm{H}$. pylori positive and the control group ( $p>0.05$ ).

There was statistically no significant difference between CRP levels, platelet counts, MPV levels and NLR of the children with $\mathrm{CU}$ with other concomitant chronic inflammatory conditions and those children with only CU $(n=27, p>0.05)$. No statistically significant difference was found in platelet counts, MPV levels and NLR between the children with $\mathrm{CU}$ with concomitant and other chronic inflammatory conditions and the control group ( $p>0.05)$.

\section{Discussion}

Many etiologic factors have been associated with the origin of $\mathrm{CU}$, but most cases are idiopathic $(1,2)$. Physical factors play a major role in CU (1). Of our children with $\mathrm{CU}$, IU made up $23.9 \%$ and CSU accounted for $76.1 \%$.

Table I. The laboratory values of the chronic urticaria and control groups

\begin{tabular}{|l|l|l|l|}
\hline & $\begin{array}{l}\text { Chronic } \\
\text { urticaria, } \\
\mathbf{n = 4 6}\end{array}$ & $\begin{array}{l}\text { Control } \\
\text { group, } \mathbf{n = 3 0}\end{array}$ & p value \\
\cline { 2 - 3 } & Mean \pm SD & Mean \pm SD & \\
\hline Leukocyte, (103/uL) & $7.3 \pm 2.1$ & $7.8 \pm 1.9$ & 0.254 \\
\hline Hemoglobin, (g/dL) & $13.3 \pm 1.1$ & $13.1 \pm 1.3$ & 0.375 \\
\hline Neutrophil, (103/uL) & $3.9 \pm 1.6$ & $4.3 \pm 1.6$ & 0.186 \\
\hline Lymphocyte, (103/uL) & $2.6 \pm 0.8$ & $2.6 \pm 0.7$ & 0.973 \\
\hline NLR & $1.6 \pm 0.8$ & $1.9 \pm 1.1$ & 0.135 \\
\hline Platelet, (103/uL) & $285.9 \pm 70.2$ & $316.3 \pm 93.0$ & 0.109 \\
\hline MPV, (fL) & $8.9 \pm 1.4$ & $9.2 \pm 1.2$ & 0.427 \\
\hline $\begin{array}{l}\text { SD: Standard deviation, NLR: Neutrophil-to-lymphocyte ratio, MPV: Mean } \\
\text { platelet volume }\end{array}$ & \\
\hline
\end{tabular}

The pathogenetic mechanism of $\mathrm{CU}$ is not yet well understood; however, the activation of skin mast cells play a key role in inflammation associated with the disease. Mast cell activation and the release of mediators, particularly histamine, can impact cutaneous inflammatory processes and the accumulation and activation of other cells $(4,13,14)$. The activation and degranulation of mast cells can be triggered by a number of mechanisms such as autoimmunity, complement components, and coagulation cascade $(4,6)$. In recent years, platelets have been found to play a dominant role in the immune/inflammatory processes (15). The role of platelets in inflammatory skin diseases such as atopic dermatitis, psoriasis, and urticaria has been evaluated $(15,16)$. It is considered that activated platelets might have an impact on mast cells, probably through the activation of the coagulation cascade $(8,16)$. It has also been reported that platelet activation products such as platelet factor 4 and beta-thromboglobulin levels are elevated in pressure and cold urticaria which are subtypes of IU $(17,18)$.

CRP is a sensitive systemic marker of inflammation, responding to interleukin-6 (IL-6) (7). In recent years, some systemic inflammatory diseases have been shown to be positively associated with CRP (19-22). In our study, there was no difference in CRP levels between the CSU and IU groups. Since the CRP could not be examined in the healthy children in our study, we are unable to make any further comment.

MPV shows platelet size and it is used to show platelet function and activation. While the count of platelets increases during inflammation, their volume tends to decrease or increase (23). MPV increases in type 2 DM, smoking, hypertension, hypercholesterolemia, obesity, coronary heart diseases and metabolic syndrome (12). In our study, only one patient (2.2\%) had an increased MPV (MPV $>12.0 \mathrm{fL}$ ). No additional pathology was found to affect MPV levels in the children with CU. In our study, MPV levels and platelet counts showed no difference between the CSU, $\mathrm{IU}$, and control groups. In Kasperska-Zając et al. (22) study, no difference was found in the platelet counts and MPV levels between CSU and healthy control subjects. When the platelet counts were evaluated according to disease severity, in the severe group, it was found to be significantly higher than the less severe group and the control group. While Isiksacan et al. (24) found low MPV, Confino-Cohen et al. (25) found high MPV in CU. Akelma et al. (11), though, found the MPV value to be lower in the CSU group than the control group, and the platelet counts to be higher, and they reported that the decline in MPV may be used as an inflammatory marker in pediatric patients with CSU. 
For CSU patients resistant to histamine, no difference was found in their platelet counts compared to control and non-histamine resistant CSU, while the MPV values were determined as higher (20). Additionally, higher MPV levels were determined in patients with $\mathrm{CU}$ using the positive autologous serum skin test (26-28). In the literature, these different results suggested to us that more studies need to be performed with a larger volume of patients. Different results may be associated with patients being in different age groups [only Akelma et al. (11) study population is based on a children's group, the other studies are focused on adult age groups $(20,22,24-28)]$ and also the patients being grouped in different ways may cause results to differ. In addition, because our patient group was mainly made up of the childhood age group, the severity of the disease, and the correlation between the severity of the disease and the inflammation parameters could not be evaluated.

NLR is a cost-effective method, and high levels of NLR are considered as an inflammatory marker (29). We have not encountered any studies in our literature review that investigated NLR being used as an inflammatory marker in urticaria yet. In our study, no difference was found in NLR levels among children with $\mathrm{CU}$ and healthy children, and the CSU group and the IU group. In studies of psoriasis and FMF, high NLR was found and it was reported that NLR is a marker of inflammation in patients with psoriasis and FMF $(9,10,30,31)$.

We did not find any differences in the terms of MPV levels and NLR among children with CU with $H$. pylori positive, children with $\mathrm{CU}$ with $H$. pylori negative and the control group. Additionally we also did not find any differences in the terms of MPV levels and NLR among children with $\mathrm{CU}$ with concomitant and other chronic inflammatory conditions, only children with CU and control group. These findings show us that MPV and NLR do not change in chronic inflammation. Also, according to these findings, we did not think concomitant and other inflammatory conditions led to a bias.

\section{Study Limitations}

The greatest limitation of our study was that it was a retrospectively designed study so there was an inability to show CRP in healthy children, to show disease severity, and there was an inability to get the results of $H$. pylori for all children with CU.

\section{Conclusion}

In our study, we detected that the platelet count, MPV levels, and the NLR did not differ in children with CU, CSU, and IU and the control group. It seems that the simple tests like MPV and NLR cannot be used as inflammatory markers of $\mathrm{CU}$ in children. We believe that more studies need to be performed pertaining to this topic.

\section{Ethics}

Ethics Committee Approval: Retrospective study.

Informed Consent: An informed consent form was obtained from the patients' parents.

Peer-review: Externally and internally peer-reviewed.

\section{Authorship Contributions}

Concept: M.A.D., H.D., H.N.S.D., Design: M.A.D., H.D., Data Collection or Proces-sing: M.A.D., H.D., I.O.T., Analysis or Interpretation: M.A.D., H.D., Literature Search: M.A.D., H.D., I.O.T., H.N.S.D., Writing: M.A.D., H.D.

Conflict of Interest: None of the authors had conflict of interest.

Financial Disclosure: The authors declared that this study received no financial support.

\section{References}

1. Caffarelli C, Cuomo B, Cardinale F, et al. Aetiological factors associated with chronic urticaria in children: A systematic review. Acta Derm Venereol 2013;93:268-72.

2. Greenberger PA. Chronic urticaria: New management options. World Al-lergy Organ I 2014;7:31.

3. Zuberbier $T$, Aberer W, Asero R, et al. The EAACI/GA(2) LEN/ EDF/WAO Guideline for the definition, classification, diagnosis, and management of urticaria: The 2013 revision and update. Allergy 2014;69:868-87.

4. Maurer M, Church MK, Gonçalo M, Sussman G, Sánchez-Borges M. Management and treatment of chronic urticaria (CU). J Eur Acad Dermatol Venereol 2015;29(Suppl 3):16-32.

5. Mlyneka A, Maurerb M, Zalewskaa A. Update on chronic urticaria: Focusing on mechanisms. Curr Opin Allergy Clin Immunol 2008;8:433-7.

6. Jain S. Pathogenesis of chronic urticaria: An overview. Dermatol Res Pract 2014;2014:674709.

7. Kasperska-Zajac A. Acute-phase response in chronic urticaria. I Eur Acad Dermatol Venereol 2012;26:665-72.

8. Vena GA, Cassano N, Marzano AV, Asero R. The role of platelets in chronic urticaria. Int Arch Allergy Immunol 2016;169:71-9.

9. Sen BB, Rifaioglu EN, Ekiz O, Inan MU, Sen T, Sen N. Neutrophil to lymphocyte ratio as a measure of systemic inflammation in psoriasis. Cutan Ocul Toxicol 2014;33:223-7.

10. Özer S, Yılmaz R, Sönmezgöz E, et al. Simple markers for subclinical inflammation in patients with Familial Mediterranean Fever. Med Sci Monit 2015;21:298-303.

11. Akelma AZ, Mete E, Cizmeci MN, Kanburoglu MK, Malli DD, Bozkaya $\mathrm{D}$. The role of mean platelet volume as an inflammatory marker in children with chronic spontaneous urticaria. Allergol Immunopathol (Madr) 2015;43:10-3. 
12. Vizioli L, Muscari S, Muscari A. The relationship of mean platelet volume with the risk and prognosis of cardiovascular diseases. Int I Clin Pract 2009;63:1509-15.

13. Ferrer M. Immunological events in chronic spontaneous urticaria. Clin Transl Allergy 2015;5:30.

14. Hennino A, Bérard F, Guillot I, Saad N, Rozières A, Nicolas JF. Pathophysiology of urticaria. Clin Rev Allergy Immunol 2006;30:311.

15. Katoh N. Platelets as versatile regulators of cutaneous inflammation. J Dermatol Sci 2009;53:89-95.

16. Katayama I, Matsui S, Murota H. Platelet activation as a possible indicator of disease activity in chronic urticaria: Link with Blood Coagulation and Mast Cell Degranulation. J Clin Exp Dermatol Res 2013;4:194.

17. Grandel KE, Farr RS, Wanderer AA, Eisenstadt TC, Wasserman SI. Association of platelet-activating factor with primary acquired cold urticaria. N Engl J Med 1985;313:405-9.

18. Kasperska-Zajac A, Brzoza Z, Rogala B. Increased concentration of platelet-derived chemokines in serum of patients with delayed pressure urticaria. Eur Cytokine Netw 2008;19:89-91.

19. Canpolat F, Akpinar H, Eskioğlu F. Mean platelet volume in psoriasis and psoriatic arthritis. Clin Rheumatol 2010;29:325-8.

20. Magen E, Mishal J, Zeldin Y, Schlesinger M. Clinical and laboratory features of antihistamine-resistant chronic idiopathic urticaria. Allergy Asthma Proc 2011;32:460-6.

21. Güneş $A$, Ece $A$, Şen $V$, et al. Correlation of mean platelet volume, neutrophil-to-lymphocyte ratio, and disease activity in children with juvenile idiopathic arthritis. Int / Clin Exp Med 2015;8:11337-41.

22. Kasperska-Zając A, Grzanka A, Jarzab l, et al. The association between platelet count and acute phase response in chronic spontaneous urticaria. Biomed Res Int 2014;2014:650913.
23. Gasparyan AY, Ayvazyan L, Mikhailidis DP, Kitas CD. Mean platelet volume: A link between thrombosis and inflammation? Curr Pharm Des 2011;17:47-58.

24. Isiksacan N, Koser M, Cemsitoglu F, Kucuksezer UC, Gurdol F. Platelet and other hemostatic characteristics in patients with chronic urticaria. Angiology 2015;66:387-91.

25. Confino-Cohen R, Chodick G, Shalev V, Leshno M, Kimhi O, Goldberg A. Chronic urticaria and autoimmunity: Associations found in a large population study. I Allergy Clin Immunol 2012;129:1307-13.

26. Magen E, Mishal J, Zeldin $Y$, et al. Increased mean platelet volume and C-reactive protein levels in patients with chronic urticaria with a positive autologous serum skin test. Am I Med Sci 2010;339:504-8.

27. Aleem S, Masood Q, Hassan I. Correlation of mean platelet volume levels with severity of chronic urticaria. I Dermatol Dermatol Surg 2015;19:9-14.

28. Chandrashekar L, Rajappa M, Sundar I, et al. Platelet activation in chronic urticaria and its correlation with disease severity. Platelets 2014;25:162-5.

29. Imtiaz F, Shafique K, Mirza SS, Ayoob Z, Vart P, Rao S. Neutrophil lymphocyte ratio as a measure of systemic inflammation in prevalent chronic diseases in Asian population. Int Arch Med 2012;5:2.

30. Ataseven A, Bilgin AU, Kurtipek GS. The importance of neutrophil lymphocyte ratio in patients with psoriasis. Mater Sociomed 2014;26:231-3.

31. Ünal $M$, Küçük $A$, Ünal $G U ̈$, et al. Mean platelet volume, neutrophil to lyphocyte ratio and platelet to lymphocyte ratio in psoriasis. Turkderm 2015;49:112-6. 